\title{
Methods section remake
}

\author{
Nature Methods follows in the footsteps of Nature by ushering in an Online Methods \\ section, fully integrated with the paper, for all original research articles.
}

Even the most casual reader of the print copy of Nature Methods this month will probably notice that something is missing. The Methods section of every original research manuscript now contains only a short notice informing readers that the full methods section is available online. At first blush this seems strange for a journal whose mission is to communicate new scientific methods, but we are convinced this change offers compelling advantages, and we hope our authors and readers agree.

Previously, the length limits for articles in Nature Methods forced many authors to split the description of the methods between the Methods section in the paper and online Supplementary Methods. We always tried to ensure that the most important methods were in the main paper, but this was not always possible because of space constraints. For a journal with 'methods' in its title this was not an optimal solution.

Nature Methods authors will now be able to include a full description of the methods in a larger-roughly 3,000 words-Online Methods section that appears at the end of the PDF and HTML versions of the paper online. This change affects Articles, Brief Communications, Analyses and Resources published in Nature Methods. Other Nature Research Journals will include implementations suited to each journal.

With this change, we ask that authors prepare this section with the same justly deserved attention to detail as the rest of the manuscript. Also, unlike supplementary material, the Online Methods are copyedited for style and to help ensure their quality and consistency with the rest of the manuscript. To help facilitate this process, we ask that equation-heavy methods sections, details of chemical syntheses and methods used only to obtain supplementary data continue to be presented in supplementary material as in the past.

The implementation of Online Methods provides our authors and readers with advantages beyond consolidating most of the methods in one place. Reference lists in our papers have-out of necessity - been relatively short. This change allows the reference list to be supplemented with additional references in the Online Methods section. These will be indexed by online databases, and we encourage authors to take advantage of this capability to add appropriate citations. We hope this will herald the demise of unindexed supplementary references.

Probably the biggest change that Online Methods brings is a dedicated methods section for Brief Communications. Our readers will no longer have to dig into the Supplementary Methods to find critical methodological details in these short but highimpact reports.

Nature implemented a very similar system for Online Methods over a year ago that allowed authors to include a full methods section in the online version of their paper while providing a short methods summary in the print copy. In an effort to give authors as much space as possible to describe their work-particularly in Brief Communications - we will not include a methods summary. In fact, methods are so central to any Nature Methods paper that a separate summary would likely be redundant with the information in the main text.

For new methods with complex procedural details we have often asked authors to provide a step-bystep Supplementary Protocol detailing everything a reader needs to reproduce the method. This is something we will continue to ask for when we believe it is important for our readers. The new Online Methods will not diminish this need.

We expect that our readers and authors will appreciate the advantages that Online Methods bring to Nature Methods. With this change effectively increasing the length of Nature Methods papers-and more than doubling the length of Brief Communications - our authors will have far more space to communicate their new methodologies and cite previous work. But by limiting the increase in length to the methods section we continue to emphasize the value of succinct scientific reports. The body of the paper will remain short enough that casual readers can easily obtain the important information. The details required for more in-depth understanding or reproduction of the work will be easily accessible if needed. We hope our authors and readers are as excited by this change as we are. 